\title{
David Graeber, Bullshit Jobs
}

Paris, Les Liens qui libèrent, 2018

\section{Thomas Coutrot}

\section{(2) OpenEdition}

\section{Journals}

Édition électronique

URL : http://journals.openedition.org/travailemploi/9672

DOI : $10.4000 /$ travailemploi.9672

ISSN : 1775-416X

Éditeur

DARES - Ministère du Travail

Édition imprimée

Date de publication : 2 décembre 2019

Pagination : 131-133

ISSN : 0224-4365

Référence électronique

Thomas Coutrot, « David Graeber, Bullshit Jobs », Travail et Emploi [En ligne], 160 | 2019, mis en ligne le 01 novembre 2020, consulté le 25 mars 2021. URL : http://journals.openedition.org/travailemploi/ 9672 ; DOI : https://doi.org/10.4000/travailemploi.9672 


\section{Notes DE LECTURE}

\section{Bullshit Jobs}

\section{David Graeber}

Paris, Les Liens qui libèrent, 2018, 416 p.

\section{Lu par Thomas Coutrot ${ }^{*}$}

Dans Bullshit Jobs, le regretté David Graeber soutient une thèse qui a rencontré un large écho : une part croissante et élevée des emplois n'a ni sens ni utilité et rend malades celles et ceux qui les occupent, "à l'idée qu'ils [elles] n'apportent aucun bienfait au monde » (p. 285). Il explore les facteurs économiques, politiques et sociaux qui expliquent cette prolifération des tâches inutiles, et cherche à comprendre pourquoi ces emplois sont souvent bien mieux rémunérés que d'autres emplois beaucoup plus utiles socialement comme ceux des enseignant·es ou des infirmier.ères.

D. Graeber n'était jusqu'alors pas un spécialiste des questions de travail, même si sa curiosité et ses convictions idéologiques l'avaient déjà entraîné à publier assez loin de son domaine de compétence académique initial, l'anthropologie des rapports monétaires et de la dette. À l'origine du livre, un court billet d'humeur ${ }^{1}$ publié en 2013 dans la revue anarchiste en ligne Strike, qui fit le buzz sur le Net, suscitant une avalanche de témoignages de travailleurs qui s'y reconnaissaient. Le concept de «bullshit jobs » (ou « jobs à la con ») fit ainsi le tour du monde, rencontrant visiblement l'expérience vécue par des milliers de participants au monde du travail.

D. Graeber définit les « bullshit jobs » comme « une forme d'emploi rémunéré si totalement inutile, superflue ou néfaste que même le salarié ne parvient pas à justifier son existence, bien qu'il se sente obligé de faire croire le contraire » (p. 37). La cause du phénomène ? Pour l'auteur, les boulots inutiles se multiplient non pas pour obéir à une quelconque rationalité économique, mais bien plutôt pour satisfaire la volonté de contrôle et de domination des managers, dans une logique non pas capitaliste mais féodale, où ce qui compte est de se créer « un cortège de fidèles qui permet non seulement d'étaler son faste et sa magnificence mais aussi de prodiguer un certain nombre de faveurs politiques » (p. 247).

Peut-être cette explication est-elle partielle : elle ne répond pas à l'objection classique de l'efficacité des marchés financiers qui évinceraient les managers trop

\footnotetext{
* Direction de l'animation de la recherche, des études et des statistiques (Dares).

1. Graeber D. (2013), "On the Phenomenon of Bullshit Jobs: A Work Rant", Strike [en ligne], août, n 3 : https:// strikemag.org/bullshit-jobs/, consulté le 23 septembre 2019.
} 
prodigues en emplois inutiles. D'ailleurs, dans Bureaucratie ${ }^{2}$, son ouvrage précédent, D. Graeber attribuait le phénomène de bureaucratisation générale des sociétés néolibérales à une rationalité purement économique, la création de structures administratives mondiales étant essentiellement destinée à garantir l'extraction aux investisseurs de substantiels profits. Au niveau du management du travail, on a souvent observé que la multiplication des procédures et la standardisation des tâches visaient à assurer un contrôle rigoureux des performances et à stabiliser la rentabilité du capital à un niveau historiquement élevé, en sacrifiant la capacité d'innovation de la plupart des salariés. Néanmoins, l'invocation, par l'auteur, de la logique féodale est loin d'être absurde, tant elle rejoint nombre d'observations connues sur l'inefficacité économique des méthodes contemporaines de management, obsédées par la programmation détaillée des actes de travail et leur surveillance électronique permanente ${ }^{3}$.

Sur le plan quantitatif, D. Graeber estime que le phénomène des «bullshit jobs » est d'une ampleur considérable, mais ne peut s'appuyer que sur un sondage de taille limitée réalisé par l'institut YouGov UK, d'après lequel $37 \%$ des répondants estiment que leur travail n'apporte pas « une contribution significative au monde ${ }^{4}$. Dans un article, j'ai mobilisé l'enquête Conditions de travail-Risques psychosociaux 2016 pour tenter une quantification plus sérieuse du phénomène ${ }^{5}$. À première vue, il semble plus minoritaire qu' annoncé : $5 \%$ seulement des travailleurs jugent leur travail « jamais utile aux autres », et $22 \%$ qu'il ne l'est que « parfois ». En complexifiant la définition statistique pour mieux refléter la réalité décrite par D. Graeber et ses correspondants - un emploi est bullshit si le salarié juge son travail « parfois» ou « jamais » utile aux autres, et y ressent souvent de l'ennui ou n'y éprouve pas souvent la « fierté du travail bien fait »-, j'évalue à $17 \%$ le nombre de «bullshit jobs »; celles et ceux qui les occupent, comme le prévoit l'auteur, ont une santé mentale fortement dégradée.

Parmi les métiers les plus affectés, ceux de la finance, de l'informatique et de la comptabilité, qui sont souvent évoqués dans Bullshit Jobs. Mais apparaissent également des métiers ouvriers (dans les secteurs de la mécanique, des industries de process ou du gros œuvre du bâtiment) et de service (secrétaires, agents de sécurité, caissières). Le sentiment d'inutilité et d'ennui ne concerne donc pas que des emplois qualifiés : les témoignages de l'ouvrage reflètent un biais vers les professions intellectuelles.

Stimulant et souvent amusant, le livre de D. Graeber souffre néanmoins d'un trop faible ancrage dans les sciences du travail, qui l'empêche d'avancer vers des pistes crédibles de solutions. Lorsqu'il veut expliquer pourquoi les «bullshit jobs » provoquent une souffrance psychique, l'auteur convoque un psychologue allemand,

2. Graeber D. (2015), Bureaucratie, Paris, Les Liens qui libèrent.

3. Voir par exemple Weisbord M. R. (2012), Productive Workplaces: Dignity, Meaning and Community in the 21st Century, San Francisco (CA), Jossey-Bass.

4. En anglais : "Is your job making a meaningful contribution to the world?" La traduction proposée dans l'édition française est approximative : «Votre emploi apporte-t-il quoi que ce soit d'important au monde ?»

5. Coutrot T. (2019), « Trop de “bullshit jobs” », Santé et Travail [en ligne], janvier ; http://www.sante-et-travail.fr/ trop-bullshit-jobs, consulté le 23 septembre 2019. 
Karl Groos, spécialiste du jeu chez l'enfant, qui écrivait en 1901 que « la joie d'être cause » est un facteur décisif du développement psychique, dont l'absence « ébranle les fondations mêmes de votre sentiment de soi » (p. 134). C'est certainement juste, mais on peut regretter l'absence de toute référence à l'abondante littérature contemporaine, en France comme à l'étranger, des sciences du travail qui traite du manque de reconnaissance au travail, des conflits éthiques et de l'amputation du pouvoir d'agir. Ainsi la théorie du «déséquilibre effort/récompense » de Johannes Siegrist met en cause le déficit de reconnaissance relativement aux efforts fournis. Le questionnaire scandinave COPSOQ (Copenhagen Psychosocial Questionnaire), l'un des plus utilisés en épidémiologie de la santé psychique au travail, prend en compte le « sens du travail » et le sentiment de «faire quelque chose d'important dans son travail ». La psychodynamique du travail de Christophe Dejours montre comment la construction de la santé mentale au travail repose largement sur le « jugement d'utilité » apporté par les supérieurs ou les usagers et sur le « jugement de beauté » qui provient de collègues reconnaissant le respect des règles de l'art. Enfin, la clinique du travail d'Yves Clot établit comment le «travail empêché », ce travail «ni fait ni à faire », est source de souffrance éthique et de troubles psychiques.

L'absence de références aux sciences du travail contribue sans doute à expliquer la faiblesse des propositions avancées par D. Graeber pour remédier à la situation, et qui se résument en fait à l'instauration d'un revenu de base. De nombreux spécialistes du travail préconisent pourtant une stratégie de changement social axée sur une démocratisation de l'organisation du travail et avancent des propositions concrètes qui peuvent inspirer une stratégie de lutte pour la débullshitisation du travail ${ }^{6}$. Nombre d'acteurs sociaux, s'appuyant sur les acquis des recherches sur le travail et les organisations, s'emploient à reconstruire le pouvoir d'agir des travailleurs sur les conditions et les finalités de leur travail, plutôt que d'attendre de l'État une mesure providentielle qui les libérerait de l'emprise du bullshit.

De la part d'un penseur anarchiste qui souhaite « le démantèlement total des États » (p. 387), la demande d'un revenu de base distribué par l'État peut sembler éminemment paradoxale, comme il le reconnaît d'ailleurs lui-même. Mais, plus fondamentalement, en quoi le revenu de base permettrait-il de faire reculer les «bullshit jobs »? Les partisans du revenu de base inconditionnel peineraient sans doute à expliquer comment ce dispositif pourrait remédier au déficit de reconnaissance, au sentiment d'inutilité, au manque de délibération sur ce que devrait être un travail bien fait ; le risque est bien plutôt qu'il accompagne l'individualisation croissante des activités de travail et rende plus difficile le débat collectif sur les finalités et la qualité du travail.

6. Voir par exemple Davezies P. (2014), "Individualisation of the Work Relationship: A Challenge for Trade Unions", Policy Brief ETUI, $\mathrm{n}^{\circ} 3$; Dejours C. (2015), Le Choix. Souffrir au travail n'est pas une fatalité, Montrouge, Bayard ; Laloux F. (2014), Reinventing Organizations: A Guide to Creating Organizations Inspired by the Next Stage of Human Consciousness, Brussels, Nelson Parker. 